\title{
Estudio de correlación de las variables significativas analizadas en el censo económico 2010
}

\section{Correlation Study of the significant variables analyzed in the 2010 Economic Census}

Raúl Álvarez Guale*

\begin{abstract}
Resumen
Este artículo presenta un estudio del análisis de correlación de las variables más significativas presentadas por el Instituto Nacional de Estadísticas y Censo (INEC) del Censo económico 2010. El estudio hace un enfoque utilizando las correlaciones lineales de Pearson, y se pretende eliminar las correlaciones sin sentido encontradas en este análisis por medio de correlaciones parciales.
\end{abstract}

\section{Palabras clave}

Econometría, correlación, covarianza, actividades económicas.

\begin{abstract}
This article presents a study of correlation analysis about the most significant variables presented by the National Institute of Statistics and Census of economical census in 2010. The study makes focus using the linear correlation of Pearson and it is pretended to eliminate nonsense correlations found in this analysis by the use partial correlations.
\end{abstract}

\section{Keywords}

Econometrics, correlation, covariance, economic activities.

Forma sugerida de citar: Álvarez, Raúl. 2011. Estudio de correlación de las variables significativas analizadas en el censo económico 2010. Retos 2. Julio/diciembre. Pp. 19-38.

* Docente UPS. Ingeniero en Estadística e Informática. Director del Centro de Investigaciones Económicas y Empresariales (CIEE) de la Universidad Poliécnica Salesiana. E mail: ralvarezg@ups.edu.ec.

Retos 2(I): 2011 .

(c) 20II, Universidad Politécnica Salesiana del Ecuador 


\section{Introducción}

La Econometría es el campo de la economía que se ocupa de la medición empírica tanto sean éstas como la estimación, la inferencia y las predicciones de variables; la econometría también analiza las relaciones entre variables que establece la teoría económica, a través de la aplicación de métodos estadísticos, matemáticos y computacionales. El propósito fundamental es proporcionar contenido empírico a las relaciones teóricas. (Cisepa, 2003).

Este trabajo se centra en utilizar una técnica econométrica para determinar las relaciones existentes entre las siguientes variables: Cantidad de personal que trabajan en las empresas económicamente activas, los Gastos por remuneración que tienen las empresas, el Financiamiento obtenido en todo el período de estudio, los Gastos por electricidad generados, los Ingresos netos obtenidos, y el uso de Internet.

La herramienta para determinar la relación existente entre cada una de las variables es la el coeficiente de correlación poblacional $\rho \rho$ de Pearson, que se define como:

$\rho=\frac{\operatorname{cov}(x, y)}{\sqrt{\{\operatorname{var}(x) \operatorname{var}(y)]}}=\frac{\operatorname{cov}(x, y)}{\sigma_{x} \sigma_{y}}$

Fuente: (Damodar Guajarati, 2010).

Donde la covarianza se calcula por medio de la fórmula:

$$
\operatorname{Cov}(x, y)=\frac{\sum x_{i} y_{i}}{n}-\bar{x} \bar{y}
$$

Fuente. (Damodar Guajarati, 2010).

Retos 2(I): 2011.

(C) 20II, Universidad Politécnica Salesiana del Ecuador

$$
\begin{aligned}
\bar{x} & =\frac{\sum x_{i}}{n} \\
\bar{y} & =\frac{\sum y_{i}}{n} \\
s_{y} & =\sqrt{\frac{\sum\left(\bar{y}-y_{i}\right)^{2}}{n}} \text { y } s_{x}=\sqrt{\frac{\sum\left(\bar{x}-x_{i}\right)^{2}}{n}}
\end{aligned}
$$

Fuente: (Damodar Guajarati, 2010).

El coeficiente de correlación es uno de los muchos coeficientes estadísticos para medir la relación lineal de varias variables, puede tomar valores entre -1 y 1 , siendo su signo igual al de la covarianza $(\operatorname{cov}(x, y)) .[1]$ Las correlaciones con valor absoluto 1 implican que existe una asociación matemática lineal perfecta, positiva o negativa, entre las dos variables y correlaciones iguales a 0 implican ausencia de asociación. Obviamente, las variables independientes tienen correlación 0 , pero nuevamente, la independencia es condición suficiente pero no necesaria (Madrid).

\section{Metodología}

Los datos presentados por el Instituto Ecuatoriano de Estadísticas y Censos (INCEC) sobre el censo económico 2010 son muy enriquecedores para la realización de estudios econométricos. La metodología a utilizar en esta investigación la podemos resumir en cuatro partes:

Parte 1: Clasificación de variables y obtención de datos

a) Identificación de las variables de investigación realizadas por el INEC.

b) Escoger seis variables significativas de estudio y renombrarlas (Debido a que los nombres son muy amplios se acortarán los mismos). 
c) Identificación de de las actividades económicas estudiadas por el INEC.

d) Obtener una tabla de los valores totales por país por cada una de las variables escogidas.

e) Obtener una tabla de los valores totales porcentuales por país por cada una de las variables escogidas.

Parte 2: Matriz de correlaciones

a) Obtener una matriz de correlaciones de Pearson de las 6 variables de estudio.

b) Realizar un diagrama de Iconografía de correlaciones para cada valor mayor a 0,5 .

Parte 3: Correlaciones parciales y semiparciales

a) Obtener una matriz de correlaciones de semiparciales combinadas para cada variable estática (fila de la matriz) y las otra par de variables como variables dinámicas (columna de la matriz).

b) Realizar un diagrama de Iconografía de correlaciones de semiparciales para cada dato de cada par de variables dinámicas (columna de la matriz) en la que cada valor de su correlación parcial superaron a 0,5.

Parte 4: Análisis de las actividades económicas

a) Obtener una matriz de correlaciones en donde cada actividad represente una variable y por cada columna agregar un 1 y el resto cero para que exista congruencia de valores entre variables y datos.

b) Realizar un diagrama de iconografía de correlaciones para cada dato de cada par de variables en la que cada valor de su correlación parcial superaron a 0,3.
El estudio está orientado a demostrar la existencia de relaciones de dos variables que se encuentren involucradas en relaciones multivariadas.

\section{Parte1. \\ Datos a utilizarse}

Los datos a utilizarse serán los proporcionados por el INEC y publicados en su página web.

\section{Censo Económico 2010}

El INEC entrega al país los resultados del Censo Nacional Económico (CENEC), realizado durante los meses de septiembre, octubre y noviembre de 2010, información de alta trascendencia con la que el Ecuador no contaba desde hace 30 años".[2]

Se investigaron 511.130 establecimientos en áreas urbanas que completaran los 2.000 habitantes o más, también se consideraron cabeceras cantonales que no cumplían con la cifra mencionada, e inclusive se tomaron en cuenta los corredores de vías principales consideradas como zonas económicas especiales y las grandes empresas.

La información recabada en el empadronamiento corresponde a la identificación y ubicación del establecimiento; clasificación de la actividad económica; ingresos, costos, gastos, existencias y activos fijos totales; personal ocupado y gastos en remuneraciones; financiamiento del establecimiento e información adicional del establecimiento.[3] 


\section{Variables de investigación:}

Las variables que se investigaron en el Censo Económico 2010, son las siguientes:

\begin{tabular}{|c|c|}
\hline & Variable estudiada \\
\hline 1 & Nombre y Razón Social del establecimiento. \\
\hline 2 & Dirección del establecimiento \\
\hline 3 & Año de constitución del establecimiento. \\
\hline 4 & Calificación artesanal. \\
\hline 5 & Tenencia del establecimiento propio o arrendado. \\
\hline 6 & Tipo de establecimiento (único, matriz, sucursal y auxiliar). \\
\hline 7 & RUC del establecimiento. \\
\hline 8 & Personal ocupado y gasto en remuneraciones. \\
\hline 9 & Actividad principal y secundaria del establecimiento. \\
\hline 10 & Principal bien o servicio. \\
\hline 11 & Materia prima. \\
\hline 12 & Fecha de inicio de la actividad principal. \\
\hline 13 & Comercio al por mayor y al por menor. \\
\hline 14 & Principal cliente. \\
\hline 15 & Costos, gastos, ingresos, existencia y activos fijos. \\
\hline 16 & Forma del establecimiento (jurídica y no jurídica). \\
\hline 17 & Fuentes de financiamiento. \\
\hline 18 & Requerimiento de financiamiento. \\
\hline 19 & Investigaciones o estudios de mercado. \\
\hline 20 & Gasto en consumo de energía y combustibles. \\
\hline 21 & Manejo de desechos y/o remediación ambiental. \\
\hline 22 & Actividades de investigación y desarrollo. \\
\hline 23 & Gastos en capacitación y formación. \\
\hline 24 & Uso de internet. \\
\hline 25 & Afiliación a gremios, cámaras u otras formas de asociación empresarial \\
\hline 26 & Nombre y razón social de la matriz \\
\hline 27 & Dirección de la matriz. \\
\hline 28 & Nombre del gerente y propietario. \\
\hline 29 & Datos del informante. \\
\hline 30 & Listado de sucursales. \\
\hline
\end{tabular}

Fuente: INEC-Censo Económico 2010

Elaboración: El Autor

Para este análisis de correlaciones bivariadas de variables significativas se ha escogido un total de seis por todo el país, es decir, todas y cada una de las variables que se muestran en la siguiente tabla, son valores nacionales. 


\begin{tabular}{|c|c|c|}
\hline No & Variable estudiada & $\begin{array}{l}\text { Variable estudiada } \\
\text { resumida[4] }\end{array}$ \\
\hline 1 & $\begin{array}{l}\text { Personal ocupado, según clasificación CIIU } 4.0 \text { de la actividad } \\
\text { económica principal a nivel nacional, } 2009\end{array}$ & Personal \\
\hline 2 & $\begin{array}{l}\text { Gastos en remuneraciones totales de los establecimientos úni- } \\
\text { cos y matrices censados en el } 2009 \text {, según clasificación CIIU } 4.0 \\
\text { de la actividad principal }\end{array}$ & Gastos remuneración \\
\hline 3 & $\begin{array}{l}\text { Monto de financiamiento obtenido en el } 2009 \text { de establecimien- } \\
\text { tos económicos únicos y matrices censados, según CIIU } 4.0 \text { de } \\
\text { la actividad principal nacional. }\end{array}$ & Financiamiento \\
\hline 4 & Ingresos anuales de los establecimientos. & Ingresos \\
\hline 5 & $\begin{array}{l}\text { Gastos de energía eléctrica anual de los establecimientos únicos } \\
\text { y matrices censados en el 2009; según CIIU de la actividad } \\
\text { económica principal a nivel nacional. }\end{array}$ & Gastos de energía \\
\hline 6 & $\begin{array}{l}\text { Uso del Internet en establecimientos únicos y matrices censa- } \\
\text { dos, según clasificación CIIU } 4.0 \text { de la actividad principal a nivel } \\
\text { nacional, } 2009 .\end{array}$ & Uso de Internet \\
\hline
\end{tabular}

Fuente: INEC-Censo Económico 2010

Elaboración: El Autor

\section{Actividades económicas}

El INEC se ha basado en la clasificación de las actividades económicas por medio de la Clasificación Industrial Internacional Uniforme de las Actividades Económicas (CIIU) emitido por del departamento de Asuntos Económicos y Sociales de la división Estadística de las Naciones Unidas.[5]

Esta clasificación hace una categorización de las actividades económicas productivas para que se puedan utilizar en la elaboración de estudios estadísticos. En la Tabla 1. se muestra los nombres generales de cada una de las actividades:

Tabla 1. Actividades Económicas según CIIU 4.0

\begin{tabular}{|c|l|c|}
\multicolumn{1}{|c}{ Actividades Económicas } & $\begin{array}{r}\text { Nombre Activida- } \\
\text { des Económicas } \\
\text { resumida para caso } \\
\text { de estudio }\end{array}$ \\
\hline 1 & Agricultura, ganadería, silvicultura y pesca. & A1 \\
\hline 2 & Explotación de minas y canteras. & A2 \\
\hline 4 & Industrias manufactureras. & Suministro de electricidad, gas, vapor y aire acondicionado. \\
\hline 5 & $\begin{array}{l}\text { Distribución de agua; alcantarillado, gestión de desechos y activi- } \\
\text { dades de saneamiento. }\end{array}$ & A4 \\
\hline 6 & Construcción. & A5 \\
\hline 7 & $\begin{array}{l}\text { Comercio al por mayor y al por menor; reparación de vehículos } \\
\text { automotores y motocicletas. }\end{array}$ & A6 \\
\hline 8 & Transporte y almacenamiento. & A7 \\
\hline 9 & Actividades de alojamiento y de servicio de comidas. & A8 \\
\hline
\end{tabular}


Estudio de correlación de las variables significativas analizadas en el censo económico 2010

\begin{tabular}{|c|c|c|}
\hline 10 & Información y comunicación. & A10 \\
\hline 11 & Actividades financieras y de seguros. & A11 \\
\hline 12 & Actividades inmobiliarias. & A12 \\
\hline 13 & Actividades profesionales, científicas y técnicas. & A13 \\
\hline 14 & Actividades de servicios administrativos y de apoyo. & A14 \\
\hline 15 & $\begin{array}{l}\text { Administración pública y defensa; planes de seguridad social de } \\
\text { afiliación obligatoria. }\end{array}$ & A15 \\
\hline 16 & Enseñanza. & A16 \\
\hline 17 & $\begin{array}{l}\text { Actividades de atención de la salud humana y de asistencia } \\
\text { social. }\end{array}$ & A17 \\
\hline 18 & Artes, entretenimiento y recreación. & A18 \\
\hline 19 & Otras actividades de servicios. & A19 \\
\hline 20 & Actividades de organizaciones y órganos extraterritoriales. & A20 \\
\hline
\end{tabular}

Fuente: Archivo Clasificación de actividad CIIU 4.0.pdf proporcionado por el INEC[7] y nomenclatura. Elaboración: El Autor

\section{Datos del Censo Económico 2010}

En los datos proporcionado por el INEC en su archivo Tabulados Censo Nacional Económico 2009.xlsx, se muestra los valores tabulados y resumidos por cada una de las variables investigadas clasificadas por cantones, provincias, por regiones. Para nuestro caso de estudio de las seis variables se muestra el Total por país, se lo describe de la siguiente manera:

Personal. Es el personal económicamente activo con o sin remuneración que prestó sus servicios en el año 2009 en el establecimiento económico censado.

Gastos remuneración. Es el total de gastos por remuneración por sucursales y matrices que invirtió en el año 2009 cada establecimiento económico censado.

Financiamiento. Es el total del monto de financiamiento por sucursales y matrices que obtuvieron en el año 2009 cada establecimiento económico censado.

Gastos electricidad. Es el total de gastos de electricidad por sucursales y matrices que invirtieron en el año 2009 cada establecimiento económico censado.

Ingresos. Es total de ingresos que obtuvieron en el año 2009 en el establecimiento económico censado.

Internet. Es el número de establecimientos con servicio de la Internet que poseían en el año 2009 en el establecimiento económico censado. Tabla 2. Valores de totales de las variables analizadas en el Censo Económico 2010 


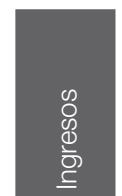

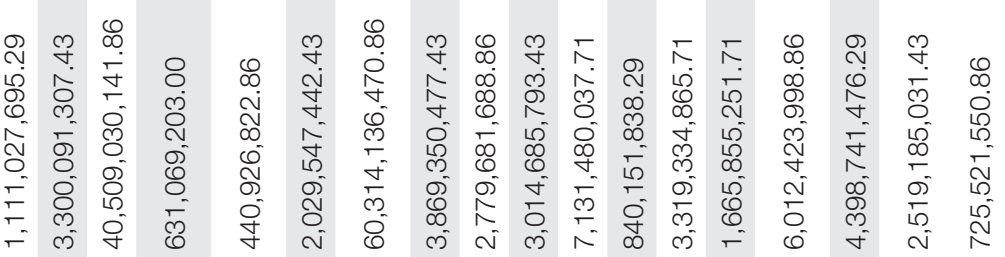

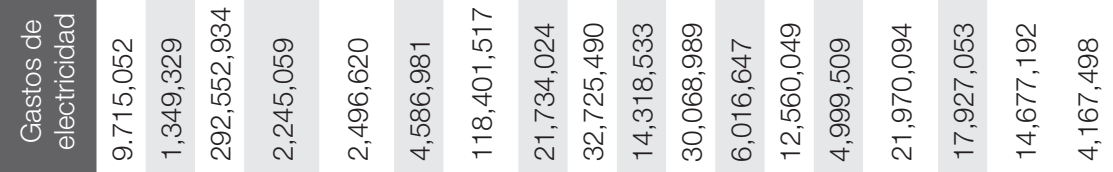

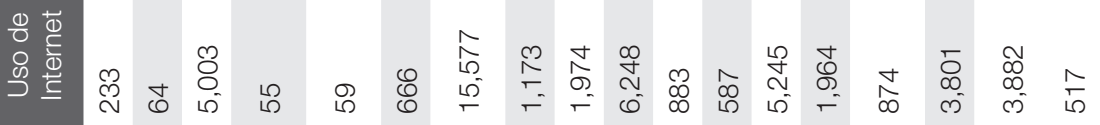

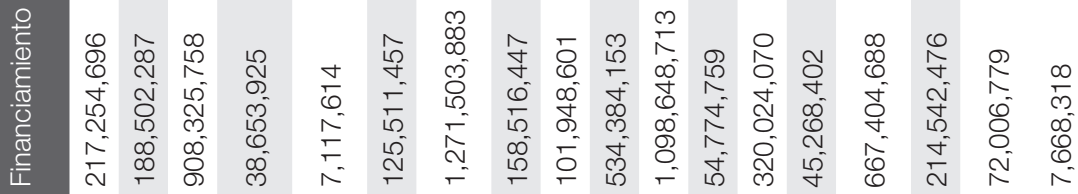

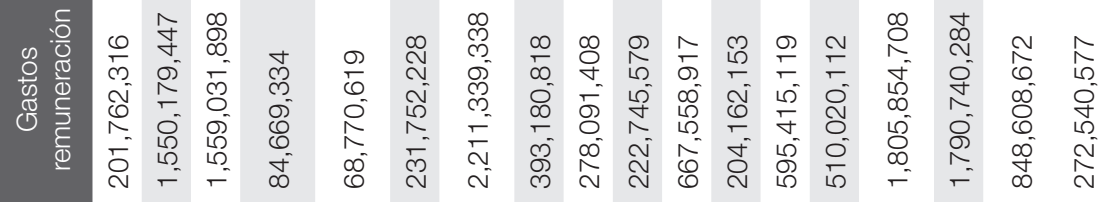

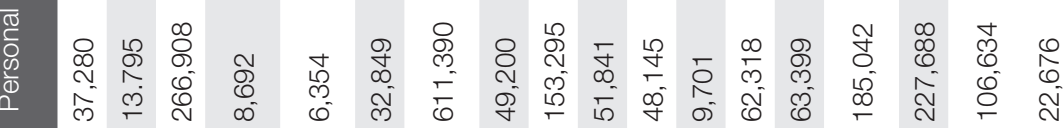

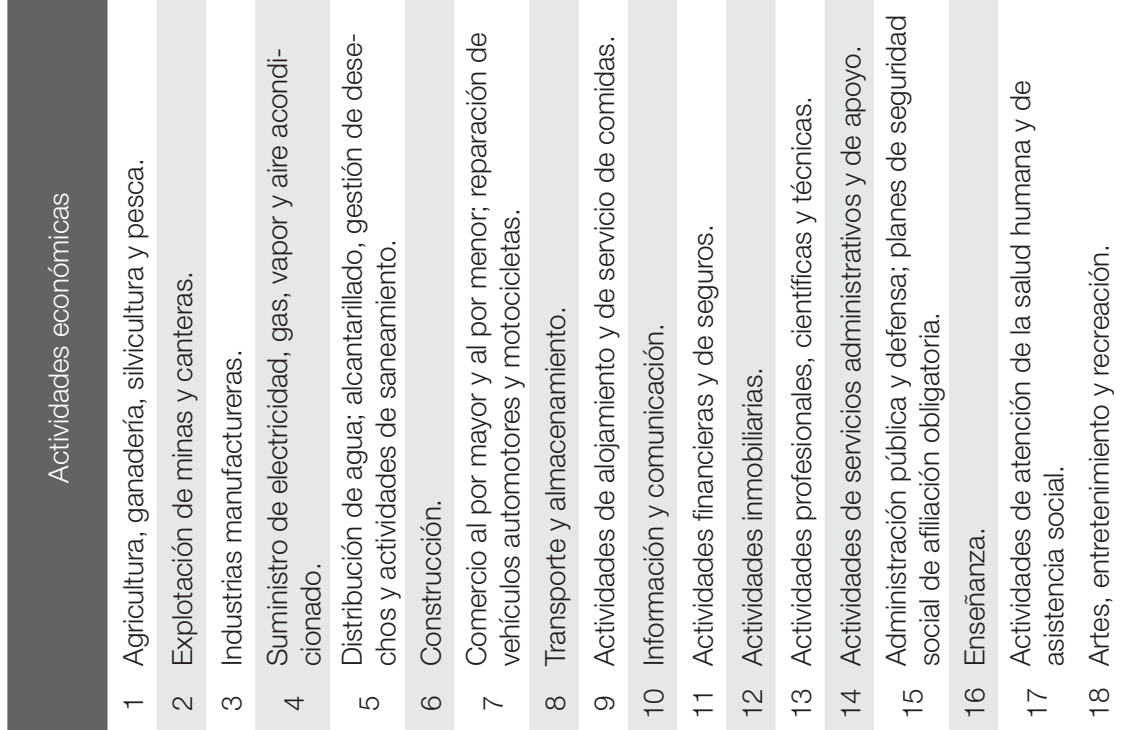

Retos 2(I): 2011.

(c) 20II, Universidad Politécnica Salesiana del Ecuador 


\section{Retos}

Estudio de correlación de las variables significativas analizadas en el censo económico 2010

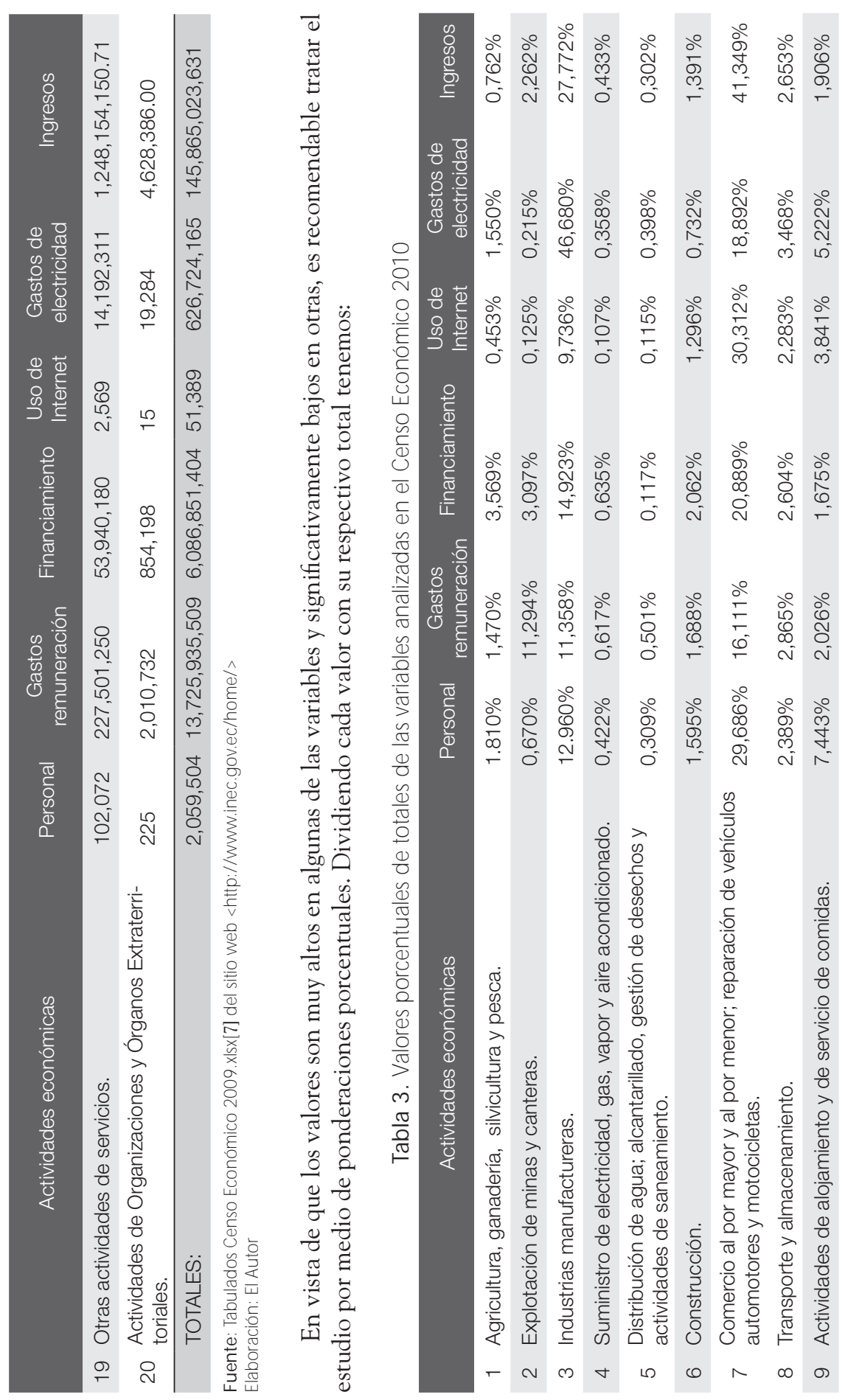

Retos 2(1): 2011.

(c) 201 I, Universidad Politécnica Salesiana del Ecuador 

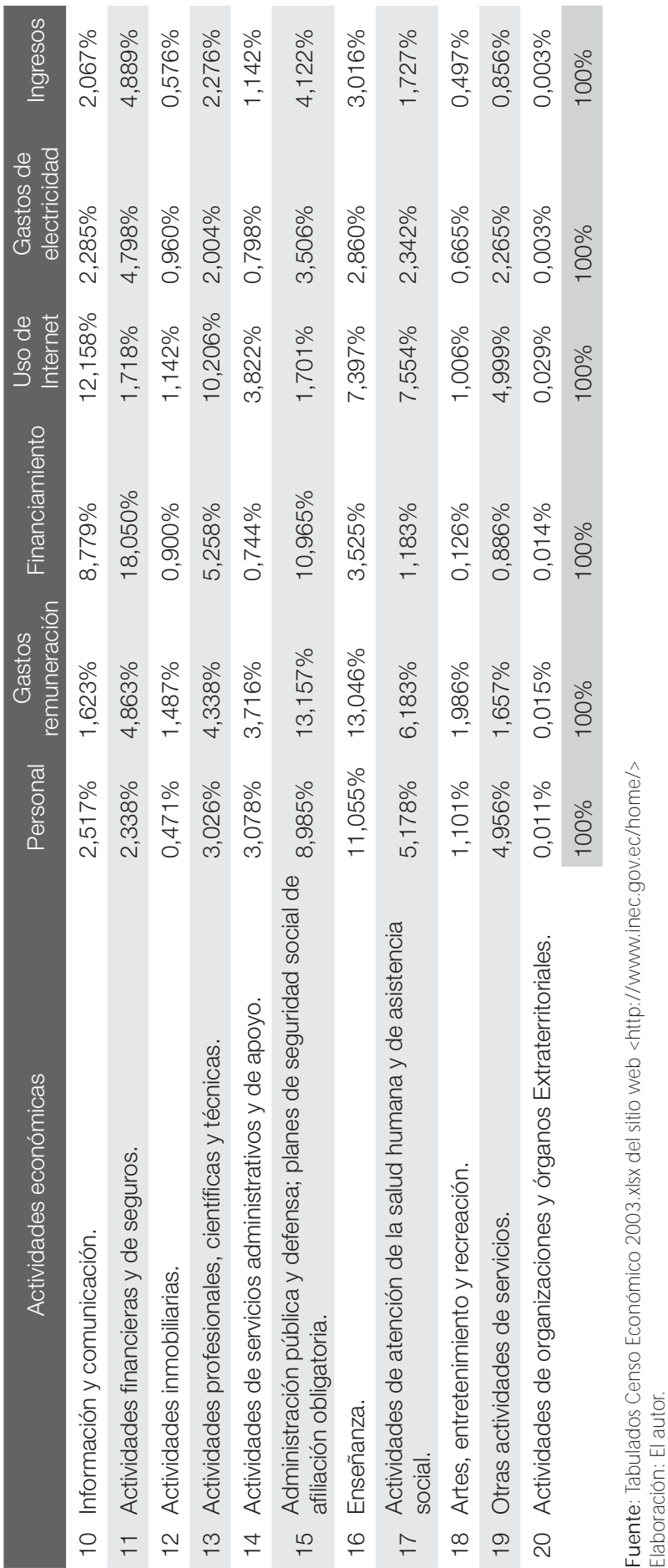

Retos 2(I): 201 I.

C) 20II, Universidad Politécnica Salesiana del Ecuador 
Estudio de correlación de las variables significativas analizadas en el censo económico 2010

Imagen 1. Porcentajes de totales PAíS de las actividades económicas y variables de estudio.

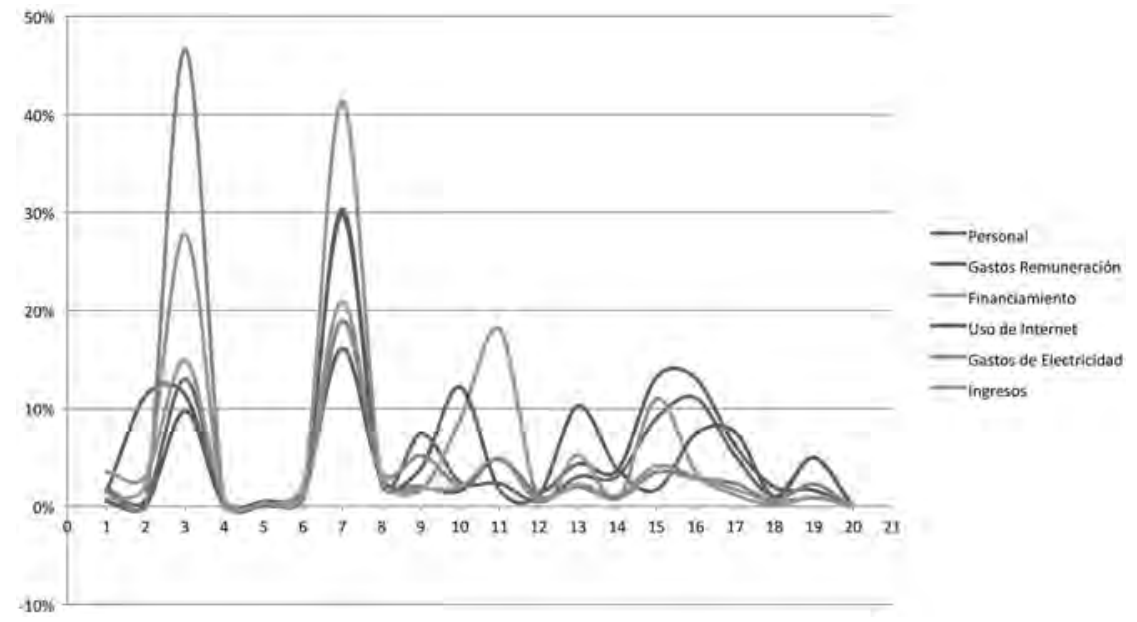

Fuente: INEC- Censo Económico 2010

Elaboración: El Autor

\section{Parte 2.}

\section{Resultados}

De acuerdo a la metodología propuesta se procede a obtener los coeficientes de correlación de Pearson entre cada una de las variables elegidas y por rama de actividad para determinar su relación existente. Se procede a obtener el coeficiente de correlación para cada par de variables. Como ejemplo se muestra el Anexo 1.

El lector podrá observar que habría que realizar cada posible combinación por cada par de variables. Para evitar este cálculo matemático se muestra el resumen de la tabla de correlaciones obtenidas por el programa estadístico SPSS.

Tabla 4. Correlación de Pearson obtenida por SPSS.

\begin{tabular}{|c|c|c|c|c|c|c|c|}
\hline & & Personal & Gastos & Financiamiento & Internet & Energía & Ingresos \\
\hline \multirow{3}{*}{ Personal } & $\begin{array}{l}\text { Pearson } \\
\text { Correlation }\end{array}$ & 1 & $0,766^{\star \star}$ & $0,693^{\star \star}$ & $0,868^{\star *}$ & $0, .597^{\star \star}$ & $0, .897^{\star *}$ \\
\hline & $\begin{array}{l}\text { Sig. } \\
\text { (2-tailed) }\end{array}$ & & 0,000 & 0,001 & 0,000 & 0,005 & 0,000 \\
\hline & $\mathrm{N}$ & 20 & 20 & 20 & 20 & 20 & 20 \\
\hline \multirow{3}{*}{ Gastos } & $\begin{array}{l}\text { Pearson } \\
\text { Correlation }\end{array}$ & $0,766^{\star \star}$ & 1 & $0,641^{\star \star}$ & $0,564^{\star *}$ & $0,496^{*}$ & $0,672^{\star \star}$ \\
\hline & $\begin{array}{l}\text { Sig. } \\
\text { (2-tailed) }\end{array}$ & 0,000 & & 0,002 & 0,010 & 0,026 & 0,001 \\
\hline & $\mathrm{N}$ & 20 & 20 & 20 & 20 & 20 & 20 \\
\hline
\end{tabular}




\begin{tabular}{|c|c|c|c|c|c|c|c|}
\hline & & Personal & Gastos & Financiamiento & Internet & Energía & Ingresos \\
\hline \multirow{3}{*}{ Financiamiento } & $\begin{array}{l}\text { Pearson } \\
\text { Correlation }\end{array}$ & $0,693^{\star *}$ & $0,641^{\star *}$ & 1 & $0,646^{\star *}$ & $0,617^{\star \star}$ & $0,781^{\star *}$ \\
\hline & $\begin{array}{l}\text { Sig. } \\
\text { (2-tailed) }\end{array}$ & 0,001 & 0,002 & & 0,002 & 0,004 & 0,000 \\
\hline & $\mathrm{N}$ & 20 & 20 & 20 & 20 & 20 & 20 \\
\hline \multirow{3}{*}{ Internet } & $\begin{array}{l}\text { Pearson } \\
\text { Correlation }\end{array}$ & $0,868^{\star \star}$ & $0,564^{\star \star}$ & $0,646^{\star *}$ & 1 & $0,473^{*}$ & $0,821^{* *}$ \\
\hline & $\begin{array}{l}\text { Sig. } \\
\text { (2-tailed) }\end{array}$ & 0,000 & 0,010 & 0,002 & & 0,035 & 0,000 \\
\hline & $\mathrm{N}$ & 20 & 20 & 20 & 20 & 20 & 20 \\
\hline \multirow{3}{*}{ Energía } & $\begin{array}{l}\text { Pearson } \\
\text { Correlation }\end{array}$ & $0,597^{\star \star}$ & $0,496^{*}$ & $0,617^{\star \star}$ & $0,473^{*}$ & 1 & $0,791^{\star *}$ \\
\hline & $\begin{array}{l}\text { Sig. } \\
\text { (2-tailed) }\end{array}$ & 0,005 & 0,026 & 0,004 & 0,035 & & 0,000 \\
\hline & $\mathrm{N}$ & 20 & 20 & 20 & 20 & 20 & 20 \\
\hline \multirow{3}{*}{ Ingresos } & $\begin{array}{l}\text { Pearson } \\
\text { Correlation }\end{array}$ & $0,897^{\star \star}$ & $0,672^{\star \star}$ & $0,781^{* *}$ & $0,821^{\star \star}$ & $0,791^{\star *}$ & 1 \\
\hline & $\begin{array}{l}\text { Sig. } \\
\text { (2-tailed) }\end{array}$ & 0,000 & 0,001 & 0,000 & 0,000 & 0,000 & \\
\hline & $\mathrm{N}$ & 20 & 20 & 20 & 20 & 20 & 20 \\
\hline
\end{tabular}

**. Correlation is significant at the 0.01 level (2-tailed).

*. Correlation is significant at the 0.05 level (2-tailed).

Fuente: INEC-Censo Económico 2010

Elaboración: El Autor

Bosquejando un gráfico de las correspondiente correlaciones de cada una de las variables, tomando en consideración un valor de correlación superior a 0,05, tenemos:

Imagen 2. Iconografía de variables

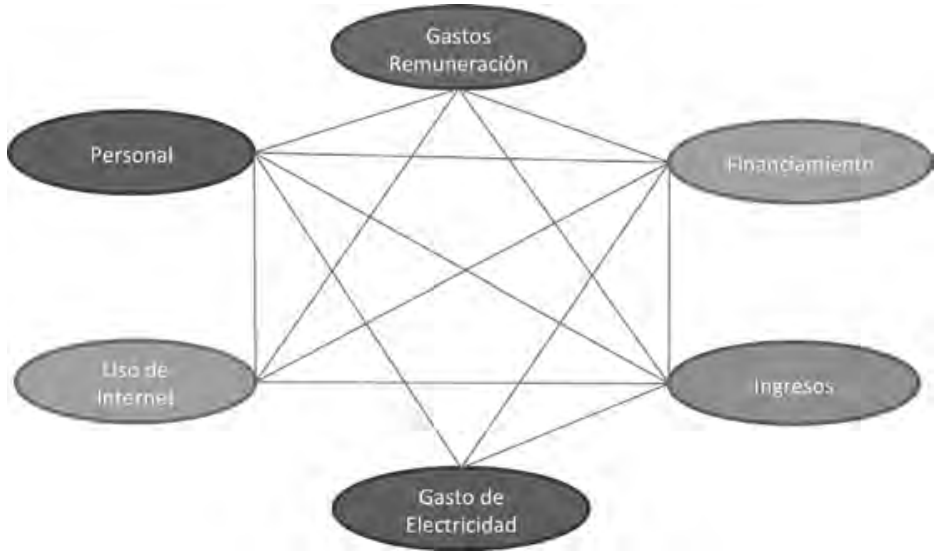

Fuente: INEC- Censo Económico 2010

Elaboración: El Autor

Retos 2(1): 2011.

C) 20II, Universidad Politécnica Salesiana del Ecuador 
Estudio de correlación de las variables significativas analizadas en el censo económico 2010

Debido a que existe mucha relación entre los porcentajes de las variables explicadas, es necesario obtener la explicación de cada una de las variables por otro método.

\section{Correlaciones parciales y} semiparciales

La correlación parcial es la correlación de una variable independiente $\left(\mathrm{X}_{\mathrm{i}}\right)$ y una variable dependiente $(Y)$ cuando se han suprimido los efectos de las otras variables independientes tanto $\mathrm{X}_{\mathrm{i}}$ e $\mathrm{Y}$ (Hair-Anderson-Tatham-Black, 2005).

Para calcular las correlaciones parciales es necesario concurrir a la siguiente fórmula:[8]

Realizando las correlaciones parciales en parejas de variables Correlación semiparcial de $Y, X_{1}$, dado $\chi_{2}$

$$
=\frac{\operatorname{Corrde} Y, X_{1}-\left(\operatorname{Corr} d e Y, X_{2} * \operatorname{Corr} \operatorname{de} X_{1}, X_{2}\right)}{\sqrt{1-\left(\operatorname{Corr} d e X_{1}, X_{2}\right)^{2}}}(6)
$$

Para tomar como ejemplo tomemos las variables:

$\mathrm{Y}=$ Personal

$\mathrm{X}_{1}=$ Gastos remuneración

$\mathrm{X}_{2}=$ Financiamiento 


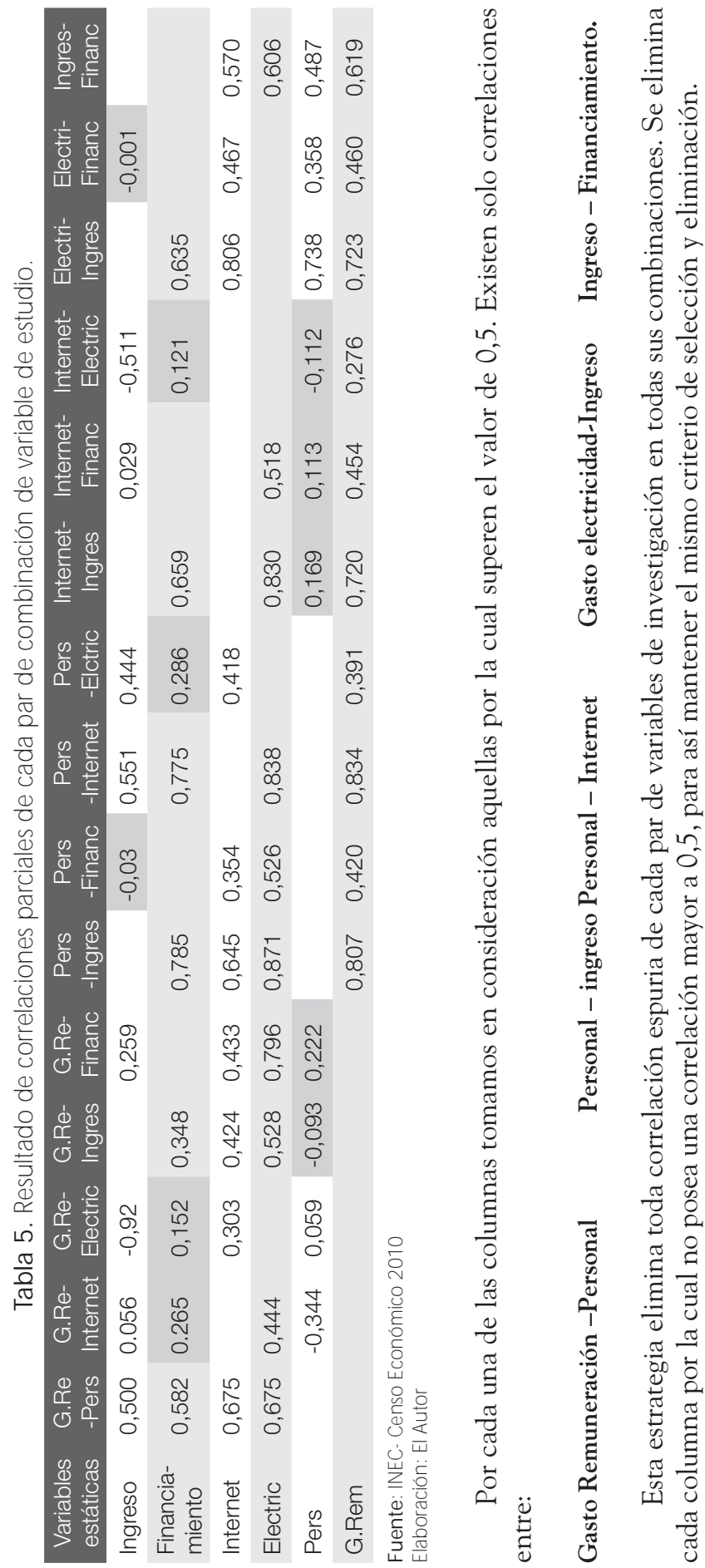

Retos 2(I): 2011.

(c) 2011, Universidad Politécnica Salesiana del Ecuador 
Estudio de correlación de las variables significativas analizadas en el censo económico 2010

Imagen 3. Correlaciones espurias eliminada para cada par de variables de investigación.

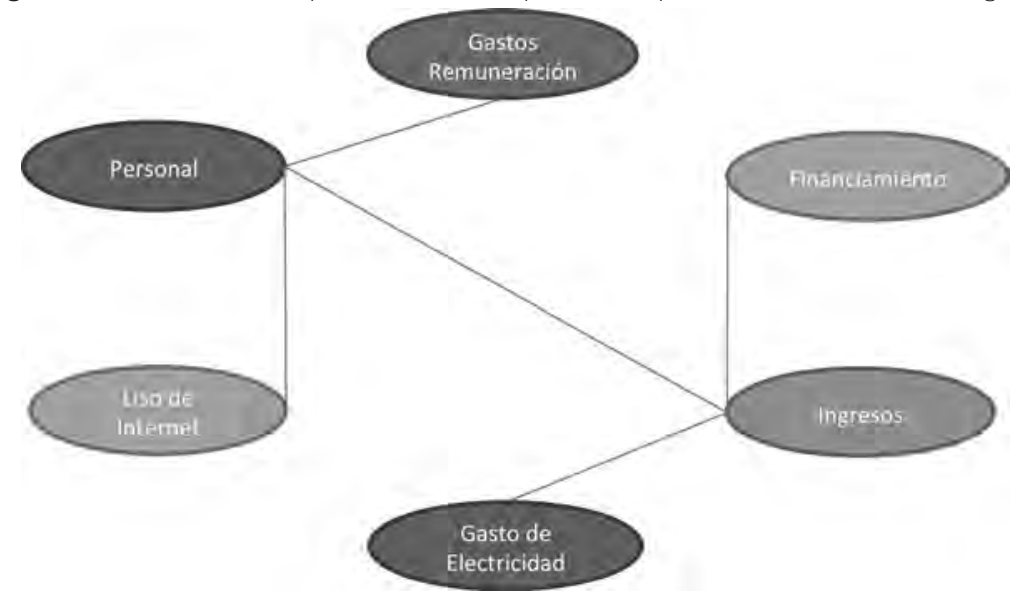

Fuente: INEC- Censo Económico 2010

Elaboración: El Autor

\section{Parte 4.}

Análisis de las actividades económicas

Los datos son favorables para permitir realizar un análisis mucho más profundo. Si bien es cierto que cada actividad económica ha representado una fila de datos de información, sería relevante saber qué o cuáles actividades económicas se encuentran aún más relacionadas con las variables de investigación, en otras palabras, qué variables pesan más con respecto a las actividades económicas, para ello debemos de considerar en efecto que cada fila se refleje como un instante del análisis, caracterizado por una variable indicadora igual a 1 en el ins- tante de la línea considerada, y a 0 , en otro caso: es decir, se considera lo siguiente:

Crear 20 columnas adicionales que reflejen las actividades económicas como variables.[9]

Se mostrará los valores de correlación mayores a 0,5 (Se sugiere también mostrar en los resultados las correlaciones mayores a 0,3)

Se adoptará el mismo criterio en donde se trazaran vínculos de relación desechadas por las correlaciones de Pearson.

Se bosquejará el gráfico resultante.

En la Tabla 6 se muestra cómo debe mostrarse la tabla para su análisis. 


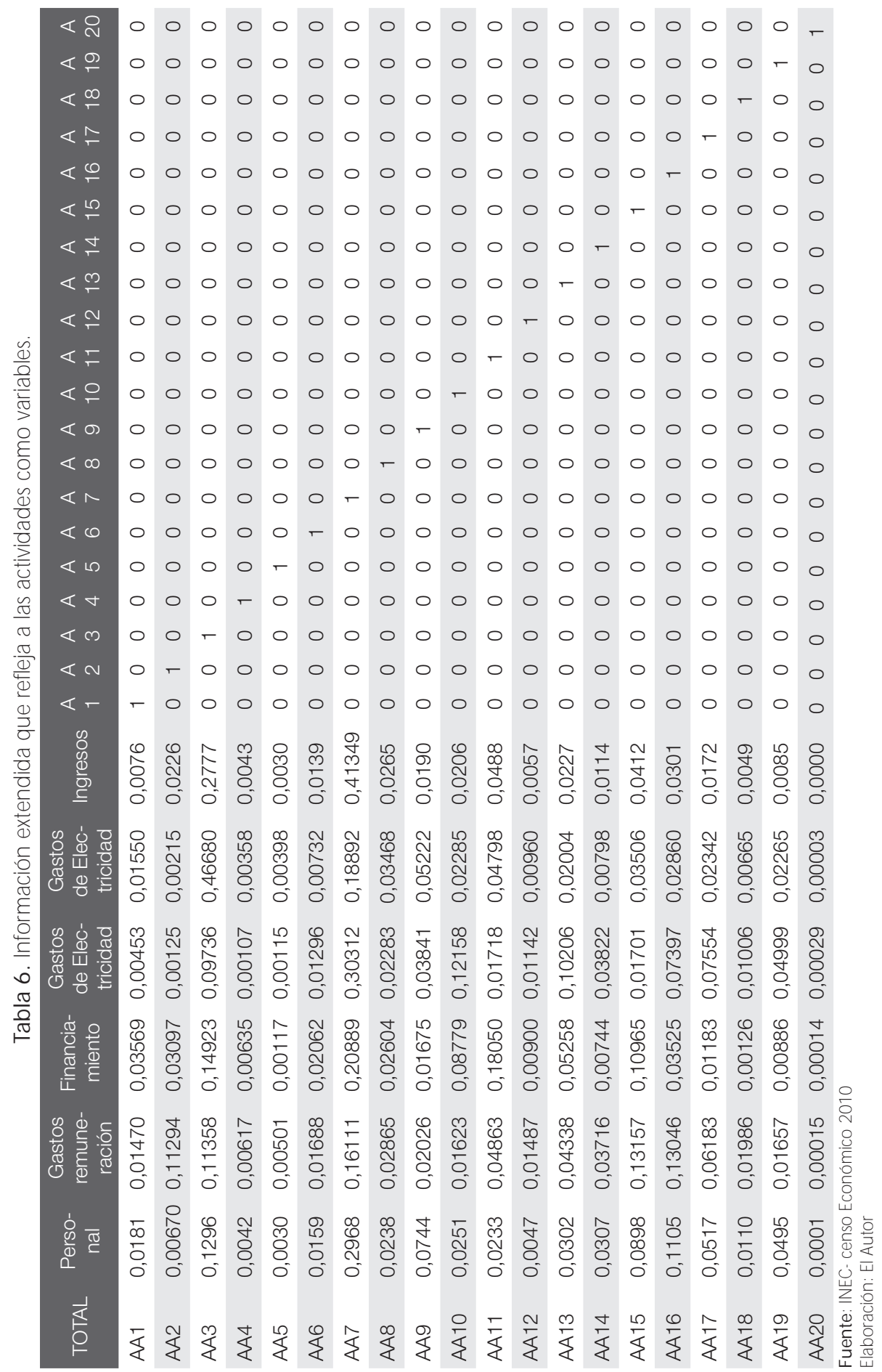

Retos 2(I): 2011.

(C) 201 I, Universidad Politécnica Salesiana del Ecuador 
Retos

Estudio de correlación de las variables significativas analizadas en el censo económico 2010

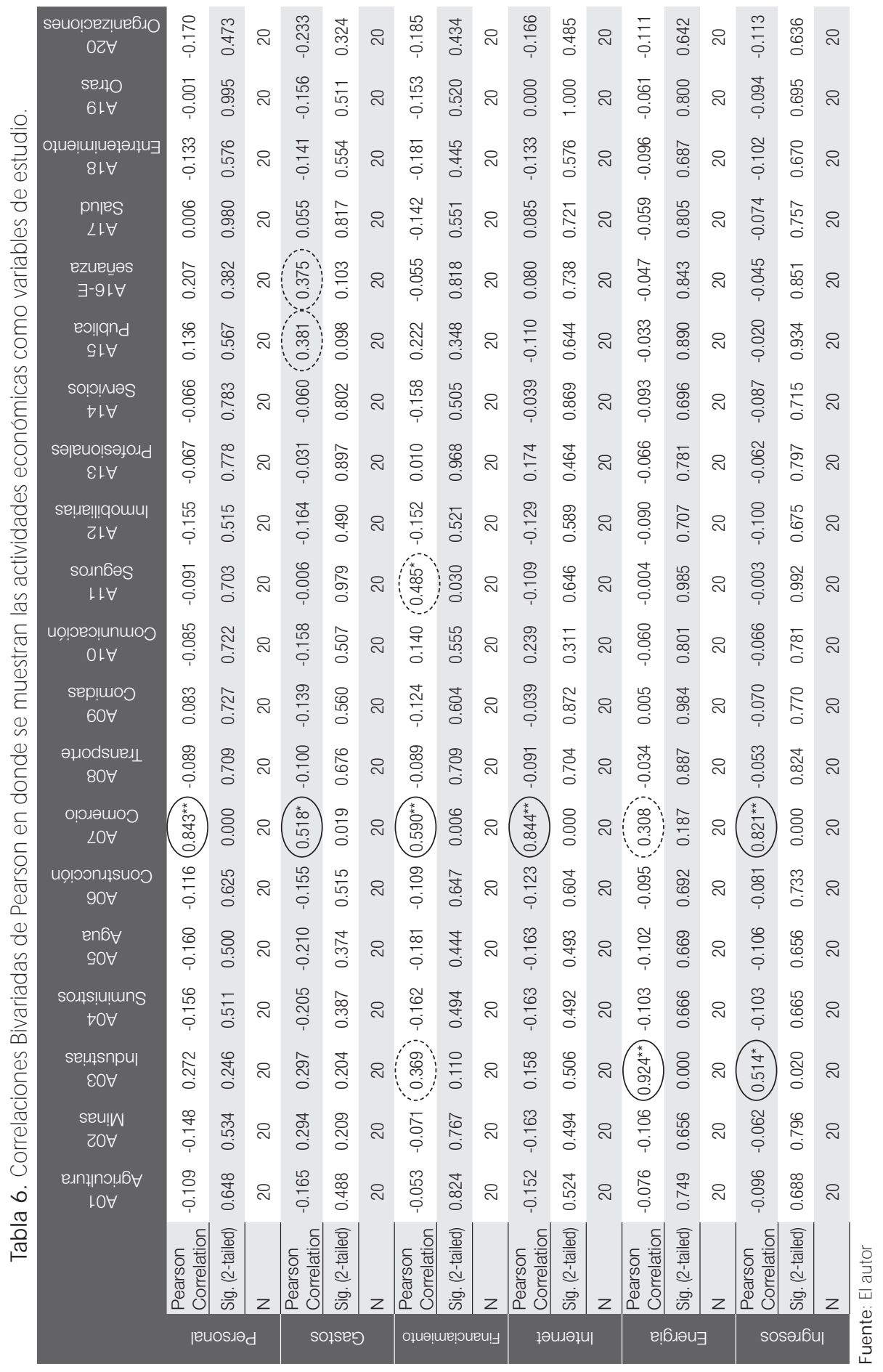

Retos 2(1): 2011.

(C) 20II, Universidad Politécnica Salesiana del Ecuador 
Realizando el gráfico correspondiente y diferenciando la actividad económica como un triángulo y la variable de investigación como un cuadrado. (M, 1999).

Imagen 4. Iconografía de Correlaciones en donde se muestran las actividades económicas con variables de investigación.

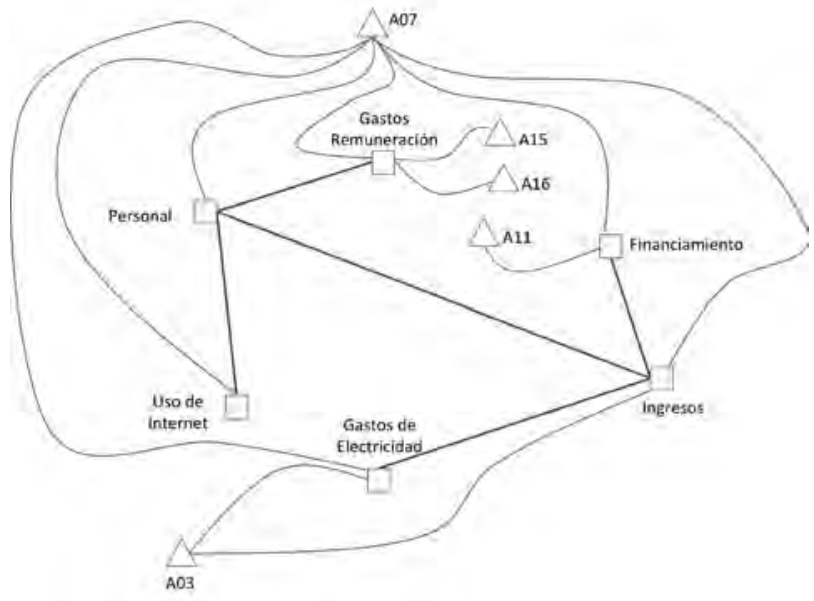

Fuente: El Autor.

\section{Conclusiones}

1. Existe una correlación lineal muy fuerte (todas mayores a 0,5), eliminado inclusive toda correlación ilegítima existente entre cada combinación de variables, siendo estas correlaciones:

- "Gastos en remuneraciones totales de los establecimientos únicos y matrices censados en el 2009, según clasificación CIIU 4.0 de la actividad principal" con el "Personal ocupado, según clasificación CIIU 4.0 de la actividad económica principal a nivel nacional, 2009”.

- El "Personal ocupado, según clasificación CIIU 4.0 de la actividad económica principal a nivel nacional, 2009" con el "Uso de la Internet en establecimientos únicos y matrices censados, según clasificación CIIU 4.0 de la actividad principal a nivel nacional, 2009".

- El personal ocupado, según clasificación CIIU 4.0 de la actividad económica principal a nivel nacional 2009 con los ingresos anuales de los establecimientos.

- El "Monto de Financiamiento obtenido en el 2009 de establecimientos económicos únicos y matrices censados, según CIIU 4.0 de la actividad principal nacional" con los "ingresos anuales de los establecimientos".

- Y los Ingresos anuales de los establecimientos con los "Gas- 
tos de energía eléctrica anual de los establecimientos úni$\cos$ y matrices censados en el 2009, según CIIU de la actividad económica principal a nivel nacional".

2. Toda variable de investigación está íntimamente correlacionada con la actividad económica "Comercio al por mayor y al por menor; reparación de vehículos automotores y motocicletas", es decir los cambios que se puedan presentar en esta actividad económica, afectan significativamente a todas la variables investigadas en este documento.

3. La actividad económica "Industrias manufactureras", está muy correlacionada a los "Gastos de energía eléctrica anual de los establecimientos únicos y matrices censados en el 2009; según CIIU de la actividad económica principal a nivel nacional" y a los "Ingresos anuales de los establecimientos".

4. Existe también una correlación significativa entre la variable "Gastos en remuneraciones totales de los establecimientos únicos y matrices censados en el 2009, según clasificación CIIU 4.0 de la actividad principal" y la actividad económica. "Administración pública y defensa; planes de seguridad social de afiliación obligatoria" y la "enseñanza".

5. Por último la variable Monto de financiamiento obtenido en el 2009 de establecimientos económicos únicos y matrices censados, según CIIU 4.0 de la actividad principal nacional" se encuentra muy correlacionada con la actividad económica "actividades financieras y de seguros".

\section{Recomendaciones}

Se debería ampliar este estudio por provincias para determinar en cuáles de ellas están más relacionadas con las actividades económicas y con las variables que se investigaron en este documento; se recomienda incluso realizarlo por cantones.

\section{Anexos}

Anexo 1: Ejemplo de las obtención del Coeficiente de Correlación de Pearson

Tabla 7. Valores porcentuales de las variables personal y Gastos en Remuneración

\begin{tabular}{cccc}
$\begin{array}{c}\text { Actividades } \\
\text { económicas }\end{array}$ & $\begin{array}{c}\text { Personal } \\
(X)\end{array}$ & $\begin{array}{c}\text { Gastos en } \\
\text { remuneración } \\
(Y)\end{array}$ & $($ XY) \\
\hline A1 & $1,810 \%$ & $1,470 \%$ & 0,00027 \\
\hline A2 & $0,670 \%$ & $11,294 \%$ & 0,00076 \\
\hline A3 & $12,960 \%$ & $11,358 \%$ & 0,01472 \\
\hline A4 & $0,422 \%$ & $0,617 \%$ & 0,00003 \\
\hline A5 & $0,309 \%$ & $0,501 \%$ & 0,00002 \\
\hline A6 & $1,595 \%$ & $1,688 \%$ & 0,00027 \\
\hline A7 & $29,686 \%$ & $16,111 \%$ & 0,04783 \\
\hline
\end{tabular}

Retos 2(1): 2011.

(C) 20II, Universidad Politécnica Salesiana del Ecuador 


\begin{tabular}{cccc|}
$\begin{array}{c}\text { Actividades } \\
\text { económicas }\end{array}$ & $\begin{array}{c}\text { Personal } \\
(X)\end{array}$ & $\begin{array}{c}\text { Gastos en } \\
\text { remuneración } \\
(\text { M }\end{array}$ & $($ XY) \\
\hline A8 & $2,389 \%$ & $2,865 \%$ & 0,00068 \\
\hline A9 & $7,443 \%$ & $2,026 \%$ & 0,00151 \\
\hline A10 & $2,517 \%$ & $1,623 \%$ & 0,00041 \\
\hline A11 & $2,338 \%$ & $4,863 \%$ & 0,00114 \\
\hline A12 & $0,471 \%$ & $1,487 \%$ & 0,00007 \\
\hline A13 & $3,026 \%$ & $4,338 \%$ & 0,00131 \\
\hline A14 & $3,078 \%$ & $3,716 \%$ & 0,00114 \\
\hline A15 & $8,985 \%$ & $13,157 \%$ & 0,01182 \\
\hline A16 & $11,055 \%$ & $13,046 \%$ & 0,01442 \\
\hline A17 & $5,178 \%$ & $6,183 \%$ & 0,00320 \\
\hline A18 & $1,101 \%$ & $1,986 \%$ & 0,00022 \\
\hline A19 & $4,956 \%$ & $1,657 \%$ & 0,00082 \\
\hline A20 & $0,011 \%$ & $0,015 \%$ & 0,00000 \\
\hline TOTALES & $100 \%$ & $100 \%$ & 0,10063 \\
\hline
\end{tabular}

Fuente: El Autor

Obtenemos los promedios de cada una de las variables;

$$
\begin{gathered}
\text { De }(3) \text { tenemos que: } \\
\bar{x}=\frac{\sum x_{i}}{n}=\frac{\mathbf{1}}{2 \mathbf{0}}=0.05 \\
\bar{x}=\frac{\sum x_{i}}{n}=\frac{\mathbf{1}}{2 \mathbf{0}}=0.05 \\
\mathrm{y} \text { de }(4) \text { obtenemos } \\
\bar{y}=\frac{\sum y_{i}}{n}=\frac{\mathbf{1}}{2 \mathbf{0}}=0.05 \\
\bar{y}=\frac{\sum y_{i}}{n}=\frac{\mathbf{1}}{2 \mathbf{0}}=0.05 \\
\operatorname{Utilizando}(2) \\
\operatorname{Cov}(x, y)=\frac{0.100630}{2 x_{i} y_{i}}-\bar{x} \bar{y}=\frac{20.05)(0.05)=0.002531}{\sigma_{x}}=0.06720 \\
\sigma_{x}=0.06720 \\
\sigma_{y}=0.04916 \\
\sigma_{y}=0.04916
\end{gathered}
$$

Finalmente utilizando (1), obtenemos[10]:

$$
\rho=\frac{\operatorname{Cov}(x, y)}{\sigma_{x} \sigma_{y}}=\frac{0.002531}{(0.06720)(0.04916)}=0.76628
$$


Estudio de correlación de las variables significativas analizadas en el censo económico 2010

\section{Notas}

[1] La covarianza mide la intensidad de variación entre dos variables

[2] Según el archivo Presentacion.pdf de la página web del El Instituto Nacional de Estadística y Censos <www.inec.gov.ec/>

[3] Según el archivo Metodología.pdf de la página web del El Instituto Nacional de Estadística y Censos <www.inec.gov.ec>

[4] Debido al largo nombre que posee la variable, se las renombrará por sugerencia del autor.

[5] Véase <http://unstats.un.org/unsd/publication/SeriesM/seriesm_4rev3_1s.pdf> página oficial de la United Nations Division.

[6] El documento fue proporcionado por funcionarios del INEC y entregado a las universidades del país por medio de un CD. También se encuentra en la página web http://www. inec.gov.ec>

[7] El documento fue proporcionado por funcionarios del INEC y entregado a las Universidades del País por medio de un CD.

[8] Se utilizó el Programa Spss para la obtención de todas las correlaciones parciales.

[9] Se ingresarán los datos en el programa SPSS para obtener los resultados de la Correlación Bivariada de Pearson.
[10] Note que el valor 0,76628 es el mismo valor de la primera fila y tercera columna de la matriz de correlaciones de Pearson.

\section{Bibliografía}

Cisepa, E. L. (2003). El coeficiente de correlación y correlaciones espúreas. Perú: Pontificia Universidad Católica del Perú, Departmento de Economía.

Damodar Guajarati, D. P., Econometría, 5ta. ed. Mexico D.C, Mc Graw Hill, 2010.

Hair-Anderson-Tatham-Black, Análisis Multivariante, 5ta. ed. Madrid, Pearson, Prentice Hall, 2005.

Vivanco, M., Muestreo Estadístico Diseño y Aplicaciones, Santiago de Chile, Editorial Universitaria, 2005.

Sitios web

$<$ http://www.inec.gov.ec/home/>

M, L. Une nouvelle approche dans le choix des régresseurs de la régression multiple en présence d'interactions et de colinéarités. Francia: Janvier.

Madrid, U. C. (s.f.). Estadística Básica. Recuperado el 1 de agosto de 2011, de <http:// www.ucm.es/info/genetica/Estadistica/estadistica_basica.htm\#coeficiente \%20de \%20 correlaci\%C3\%B3n>, 1999.

Envío 31/agosto/2011 - Aceptación 31/octubre/2011

Retos 2(1): 201 I.

(C) 2011, Universidad Politécnica Salesiana del Ecuador 